Final Technical Report for DE-FG02-08ER64700DE

\title{
Systems Level Engineering of Plant Cell Wall Biosynthesis to Improve Biofuel Feedstock Quality
}

\author{
PI: Samuel P. Hazen - University of Massachusetts \\ Co-PI: Todd C. Mockler - Donald Danforth Plant Science Center \\ Co-PI: Steve A. Kay - University of Southern California
}

\section{Mapping transcription regulatory circuits}

The goal of this project was to understand the regulatory mechanisms underlying growth and cell wall biosynthesis at the level of gene expression. The properties of the walls that surround plant cells vary dramatically across cell type and composition is an important factor determining cell shape and function. Following primary growth, some cell types construct a second thick rigid layer known as a secondary cell wall. These structures account for the bulk of renewable plant biomass and are composed of the polymers cellulose, hemicellulose, and lignin. A unique suite of enzymes assembles each class of polymer; consequently, their coordinated regulation is believed to be both complex and vital to cell function. The factors responsible for this coordinated regulation are proposed to be a hierarchy of developmental switches although the full complement of regulators remains largely unknown. My laboratory implemented a high throughput approach to

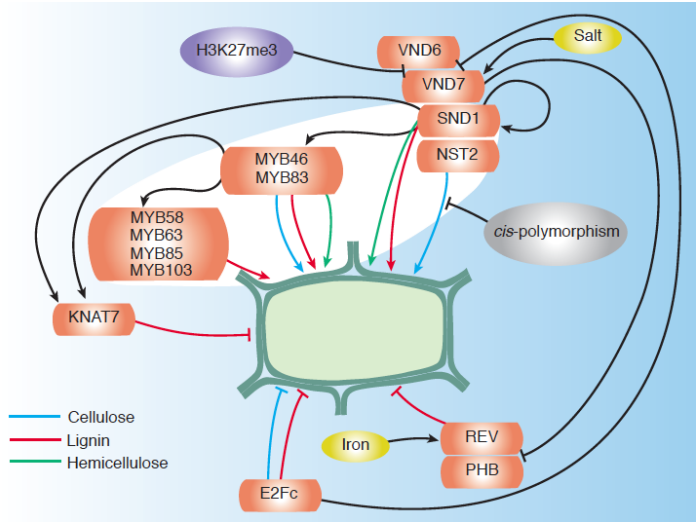

Fig 1. A non-hierarchical schematic diagram of the Arabidopsis thaliana transcriptional regulation of secondary cell wall biosynthesis. Note the prevalent direct interactions, highlighted coherent feed-forward loop, epigenetic, environmental, and natural genetic variation effects. Rectangles represent transcription factors. Ovals indicate non-protein-DNA interaction effects. The dark green line is the secondary cell wall surrounding the cell. measure direct interactions between gene promoter regions and transcription factor proteins. In collaboration with Dr. Siobhan Brady at UC Davis, we screened the promoters of A. thaliana cell wall genes and discovered something very different from the existing hierarchical model that describes mostly a series of sequential activation steps. On the contrary, we found that nearly all of the transcription factors involved, including a cell cycle regulator, directly regulate secondary cell wall metabolism genes and other transcription factors in a series of feed forward loops (Fig 1; Taylor-Teeples et al., in preparation). My laboratory also characterized a rare but naturally occurring promoter sequence polymorphism upstream of a cellulose synthesis gene that perturbs gene regulation. Impact and significance: Our new regulatory model of cell wall biosynthesis proposes original network architecture with several newly incorporated components. The mapped set of protein-DNA interactions will serve as a foundation for 1) understanding the regulation of a complex and integral plant component and 2) the manipulation of crop species for biofuel and biotechnology purposes.

The cell walls of grasses, including domesticated cereals that provide the majority of human calories and those under development as biofuel energy crops, differ significantly in 
morphology and composition from dicots such as $A$. thaliana. These salient distinctions in wall properties dictate that the flagship plant model species $A$. thaliana is unsatisfactory for understanding cell walls, and that a model grass species is required. Recently, the small grass Brachypodium distachyon (brachy) has emerged as a new model species for various food and bioenergy crops. We have completely and successfully embraced this system for all of our research. In addition to adopting many existing protocols, we have developed several of our own and laid groundwork for our studies of biomass accumulation. In order to gain vital familiarity with the species and assure proper sampling strategies and experimental design, we characterized brachy stems at various developmental stages (Matos, Whitney et al., submitted). We made the interesting observation that secondary cell walls of vascular bundle cells develop much earlier than the surrounding fiber cells. This conflicts with the doctrine that secondary wall biosynthesis uniformly synchronized at least at the transcriptional level. Impact and significance: This study revealed interesting and novel aspects of grass growth and development and further enforce the importance of a grass model system.

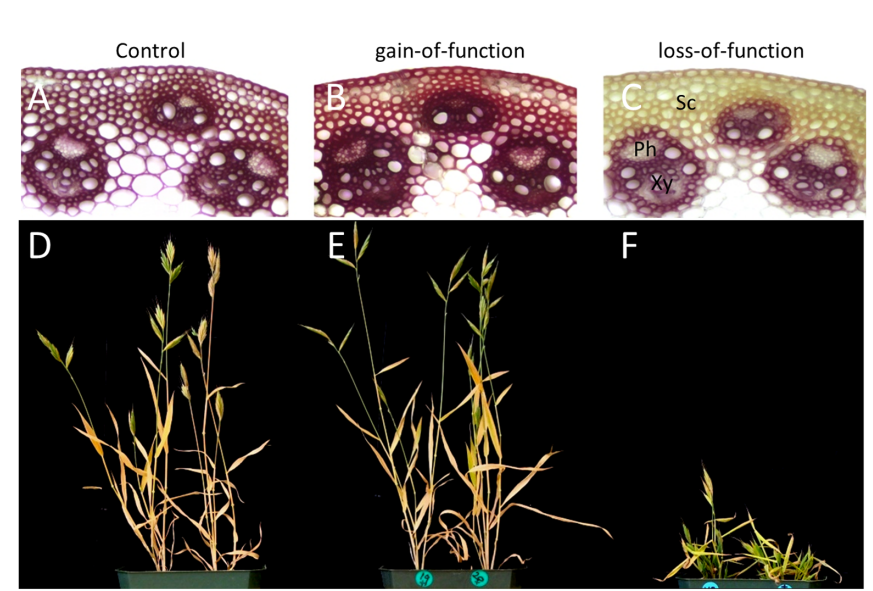

Fig 2. BdMYB48 directly controls secondary wall thickening in sclerenchyma fibers. Cross sections of stems at the first internode of developmentally equivalent plants were dissected when the flag leaf was $4 \mathrm{~cm}$ below the spike. Wiesner staining of empty vector control (A), gain-of-function Ubi::MYB48_OX (B), and loss-offunction Ubi::MYB48:CRES (C). Heavily lignified tissue stains dark red, while areas with less lignin stain orange-yellow. Representative plants of empty vector control (D), Ubi::MYB48_OX (E),

Ubi::MYB48:CRES (F). Sc, sclerenchyma fibers; $\mathrm{Ph}, \mathrm{Phloem} ; \mathrm{Xy}$, Xylem.
Again, the main focus of the project was the transcriptional regulation of cell wall biosynthesis (Handakumbura and Hazen, 2012). Because we are working with a burgeoning model system, we first demonstrated function for two lignin and two cellulose genes by characterizing brachy plants transformed with artificial microRNA predicted to target these candidates (Handakambura et al., 2013; Trabucco et al., 2013). We then set forth to characterize the proteins that directly regulate these cell wall genes. As part of the original sequencing project, my group manually annotated several transcription factor families including the sizeable MYB, NAC, MADS, and bZIP. Many of these genes serve as a foundation for a developing library of open reading frames that we used to screen for protein-DNA interactions. The characterization of several of these genes has proved to be very interesting. For example, BdMYB48 is the first grass protein shown to be a direct activator of wall genes, which it does by binding AC-type sequence elements. Gainand loss-of-function mutants have equivalent flowering times and internode number, but the loss-of-function mutant lacks secondary walls in the fiber cells that surround vascular bundles (Fig 2A). This protein may play a role in the distinct timing of wall thickening we observed when we characterized wild type stem development. Interestingly, loss-offunction mutants were more easily converted to ethanol by Clostridium phytofermentans and gain-of-function mutants exhibited significant gains in above ground biomass yield 
(Fig 3). Thus, BdMYB48 is an obvious target to engineer the improvement of biomass yield and conversion quality in energy crops.

A second gene, $B d N A C 38$, appears to be tied to both the direct regulation of cellulose biosynthesis and the transition from vegetative to reproductive growth. Unlike rosette type plants like $A$. thaliana, grasses produce stems before the developmental transition to flower. Putative flowering activators are repressed in the $B d N A C 38$ gain-of-function mutant and it persists in the vegetative phase. Our results suggest that BdNAC38 is an activator of a
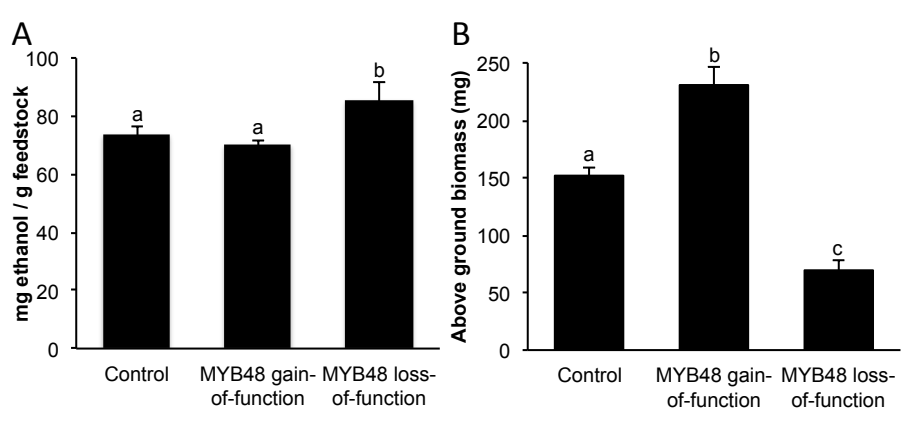

Fig 3. Biomass yield and bioconversion efficiency are altered in BdMYB48 mutant plants. (A) Transgenic loss-of-function Ubi::MYB48:CRES plants yielded more ethanol but less biomass than control and gain-of-function Ubi::MYB48_OX. Degrained plants were ground and incubated for 3 days with Clostridium phytofermentans. (B) Transgenic gain-of-function Ubi::MYB48 plants yielded more biomass that control. Data are mean \pm SEM of three separate experiments. Bars annotated with the same letter are not significantly different at $P<0.05$. flowering repressor and a direct repressor of secondary cell wall cellulose biosynthesis. Impact and significance: By functionally characterizing a suite of genes such as these, we have begun to improve the sparse model for transcription regulation of biomass accumulation in grasses. In the process, we have advanced methodology and brachy molecular genetic tools that will serve as valuable community resource.

One graduate student and four postdocs were supported by this grant. In future publications we will continue to acknowledge support that resulted in the development of numerous genetic and genomic tools.

\section{The following publications acknowledge support by Office of Science (BER) Department of Energy Grant DE-FG02-08ER64700DE.}

Handakumbura P, Hazen SP (2012) Transcriptional regulation of grass secondary cell wall biosynthesis: playing catch-up with Arabidopsis thaliana. Frontiers in Plant Science. 3:74.

Handakambura PP, Matos DA, Osmont KS, Harrington MJ, Heo K, Kafle K, Kim SH, Baskin TI, Hazen SP (2013) Perturbation of Brachypodium distachyon CELLULOSE SYNTHASE A4 or 7 results in abnormal cell walls. BMC Plant Biology. 13: 131.

Lee SJ, Warnick TA, Leschine SB, Hazen SP (2012) A high-throughput biological conversion assay for determining lignocellulosic quality. In J Normanly (ed.), Methods in Molecular Biology, vol. 918: High Throughput Phenotyping in Plants: Methods and Protocols, Springer. pp 341-349.

Lee SJ, Warnick TA, Pattathil S, Alvelo-Maurosa JG, Serapiglia MJ, McCormick H, Brown V, Young NF, Schnell DJ, Smart LB, Hahn MG, Pedersen JF, Leschine SB, Hazen SP (2012) Biological conversion assay using Clostridium phytofermentans to estimate plant feedstock quality. Biotechnology for Biofuels. 5:5. 
Trabucco GM, Matos DA, Lee SJ, Saathoff AJ, Priest HD, Mockler TC, Sarath G, Hazen SP (2013) Functional characterization of cinnamyl alcohol dehydrogenase and caffeic acid $O$ methyltransferase in Brachypodium distachyon. BMC Biotechnology. 13:61.

The following manuscripts acknowledge support by Office of Science (BER) Department of Energy Grant DE-FG02-08ER64700DE.

Li L, Pedmale UV, Schmitz RJ, Nito K, Cole BJ, Lin L, Hazen SP, Ecker JR, Chory J (submitted) PIL1 participates in a negative feedback loop that regulates its own gene expression in response to shade.

Matos DA, Whitney IP, Harrington MJ, Hazen SP (submitted) Cell walls and the developmental anatomy of the Brachypodium distachyon stem internode.

Taylor-Teeples M, Lin L Trabucco G, de Lucas M, Turco G, Toal TW, Young NF, Gaudinier A, Handakumbara PP, Breton G, Pruneda-Paz J, Ahnert S, Kay SA, Brady SM, Hazen SP (In preparation) Environmental, developmental and genotype-dependent regulation of xylem cell specification and secondary cell wall biosynthesis in Arabidopsis thaliana. 